doi:10.13108/2020-12-2-72

\title{
SECTORIAL NORMALIZATION OF SIMPLEST GERMS OF SEMI-HYPERBOLIC MAPS IN A HALF-NEIGHBORHOOD
}

\author{
P.A. SHAIKHULLINA
}

\begin{abstract}
We consider a problem on analytic classification of semi-hyperbolic maps on the plane for an example of the simplest class of germs, namely, the class of germs that are formally equivalent to $\mathrm{F}_{\lambda}$, which is the unit time shift along the vector field $x^{2} \frac{\partial}{\partial x}+\lambda y \frac{\partial}{\partial y}, \lambda \in$ $\left.\mathbb{R}_{+}\right)$. A key step in the classification is an analytic normalization of the germs on sectorial domains forming a cut neighbourhood of the origin $\left(\mathbb{C}^{2}, 0\right) \backslash\{x=0\}$. For this class, in the present work, we prove a theorem on a sectorial analytic normalization in the halfneighbourhood invariant with respect to $F_{\lambda}^{-1}$. We also show that a formal normalizing change of the coordinates is asymptotic for the constructed sectorial normalizing change.
\end{abstract}

Keywords: analytic classification, semi-hyperbolic maps, sectorial normalization.

Mathematics Subject Classifcation: 34M35, 34M40

\section{INTRODUCTION}

In work we consider a classical problem on local analytic classification of germs of homomorphisms on the plane. Let us outline briefly the history of this question.

Already in XIXth century, it was found out that as a rule, differential equations can not be solved explicitly, by quadratures. To overcome this difficulty, in the end of XIXth century, A. Poincaré proposed the following strategy of studying differential equations: if an equation can not be solved, one needs to find a change of coordinates which simplifies the form of the equation as much as possible, that is, which reduces it to a normal form. In this way, he posed two questions: what simplest form an equation can be reduced to? how to find whether it is possible to reduce one equation to another? The answers to these questions depend essentially on the classes of considered equations and changes. The same questions arise for the mappings. Both problems were solved mostly in works by Poincaré [1]-[5], Siegel [6] and Bryuno [7], [8]. Only germs of Siegel type remained unstudied under the presence of resonance or of a patological closedness to resonances [9]. In 80s of the last century essential developing were made for these special cases: in works by Yoccoz [10] for Liouville germs and in works by Voronin [11], Ecalle [12] and Martinet-Ramis [13], [14] for resonance germs.

It is turned out that in the resonance case, an obstacle for normalization of the germs is socalled functional invariants. One of the ways of constructing functional invariants is as follows [15], [16], [17]. A punctured neighbourhood of a singular point (fixed point) is covered by sectorial domains. In each of them one constructs an analytic change of coordinates normalizing the germ. The transition functions of the obtained normalizing atlas are exactly functional invariants. Thus, the problem on sectorial normalization, that is, a problem on normalization of a germ in a domain, for which the singular point (fixed point) is not internal one but is on the boundary is a first and most important step in resolving the problem on analytic classification of resonance germs.

P.A. Shaikhullina, Sectorial normalization of the simplest germs of Semi-hyperbolic maps IN A HALF-NEIGHBORHOOD.

(c) Shaikhullina P.A. 2020 .

The work is supported by RFBR (grant no. 17-01-00739a).

Submitted June 23, 2019. 
This program was completely realized for one-dimensional resonance mappings, saddle and saddle-node resonance vector fields. The next by difficulty step is two-dimensional mappings and three-dimensional vector fields.

The germs of two-dimensional mappings and three-dimensional vector fields were considered in works [16], [18] under very restrictive conditions describing the subsets of an infinite codimension in the spaces of germs. For such germs the above program was also completely realized. However, for the typical resonance mappings on the plane a little was done.

The germ at the fixed point $(0,0)$ is called semi-hyperbolic if one of its multiplicators is hyperbolic and the other is parabolic, that is, its absolute value is 1 . We note that the semihyperbolic germ is a resonance one of Siegel type. In work [19] there was obtained a semi-formal classification of such germs under some conditions for genericity.

We consider the class of germs formally equivalent to germ $\mathrm{F}_{\lambda}$ of the mapping $F_{\lambda}=\left(\frac{x}{1-x}, e^{\lambda} y\right)$, $\lambda \in \mathbb{R}_{+}$. We note that the germs considered in the work are the germs from [19] of the simplest form, we shall call them "simplest". In the present work we restrict ourselves by studying the germs in this class. We prove a theorem on sectorial normalization in a "proper" half-neighbourhood, which is invariant with respect to the inverse mapping $\mathrm{F}_{\lambda}{ }^{-1}$; the halfneighbourhood is a Cartesian product of a semi-disk and disk. In work [20], a sectorial analytical normalization of simplest germs of the class was made in a "improper" half-neighbourhood of the origin being not invariant with respect to the mapping $F_{\lambda}$ or $F_{\lambda}^{-1}$. In view of [19] and [20], this completes the first step of the above described program.

We note that even for simplest germs, the construction of sectorial normalizing mappings is non-trivial and this is why in the present work and in work [20] we consider exactly the simplest case to avoid extra calculations. However, similar arguing can be made for other formal normal forms in [19].

We should stress the following aspects. At a first glance, the construction of sectorial normalizing mappings in a "proper" half-neighbourhood invariant with respect to the mapping $F_{\lambda}^{-1}$ should not be more complicated than in the other "improper" neighbourhood. However, this is not the case since the "improper" neighbourhood contains a central manifold. We recall that a central manifold is a holomorphic submanifold tangential at the fixed point to the eigenvector of the linearization of the mapping corresponding to a non-hyperbolic multiplicator. For instance, the central manifold for $F_{\lambda}$ is $\{y=0\}$. The issue on existence of a central manifold is non-trivial. For instance, the answer to a similar question for saddle-node vector field can be formulated only in terms of Martinet-Ramis functional invariants [14. For typical semihyperbolic mappings the answer will be of course obtained once the above mentioned program is completed; this work is a part of this program. At the same time, we can pose a question on existence of a sectorial central manifold lying in a sector with the vertex at the origin. In an "improper" neighbourhood for the germs from [20] such sectorial manifold exists [19] and this simplifies essentially the problem on sectorial normalization. In the other semi-neighbourhood such central manifold not necessarily exists. A corresponding example is given by the shift in the unit time along the vector field from the known Euler example [17].

Earlier in works [21], 22, Ueda already studied the germs of semi-hyperbolic mappings and there was established the existence of a central manifold in an "improper" half-neighbourhood. In one particular case, namely, as the central manifold in such half-neighbourhood exists, in these works there were constructed sectorial normalizing mappings.

\section{Definitions AND MAIN RESUlt}

Definition 1. The germ $\mathrm{F}$ of a holomorphism $F:\left(\mathbb{C}^{2},(0,0)\right) \rightarrow\left(\mathbb{C}^{2},(0,0)\right)$ is called semihyperbolic if one of its multiplicators is equal to 1 , while the other is hyperbolic: $F(x, y)=$ $(x+\ldots, \Lambda y+\ldots)$, where $|\Lambda| \neq 0,1$. 
In particular, the germ $\mathrm{F}_{\lambda}$ of the mapping $F_{\lambda}(x, y) \mapsto\left(\frac{x}{1-x}, e^{\lambda} y\right), \lambda \in \mathbb{R}_{+}$, being the unit shift along the vector field $x^{2} \frac{\partial}{\partial x}+\lambda y \frac{\partial}{\partial y}$, is semi-hyperbolic.

As usually, two mappings $F$ and $\tilde{F}$ with different domains $U$ and $\tilde{U}$ are called analytically equivalent if there exists a holomorphic change of the coordinates $H: \tilde{U} \rightarrow U$ adjoining $\tilde{F}$ with $F:$

$$
F \circ H=H \circ \tilde{F} .
$$

At that, the equivalence is called strict if the change of coordinate $H$ in (1) reads as

$$
H(x, y)=\left(x+o\left(x^{2}\right), y+o(x)\right), \quad x \rightarrow 0 .
$$

The changes of the coordinates of such type are called normed. Two mappings are called strictly formally equivalent if there exists an invertible formal mapping $H$ of form (2), for which (1) holds as the identity of formal power series. Two germs $F$ and $\tilde{F}$ are called strictly analytically (formally) equivalent if there exist their strictly analytically (formally) equivalent representatives $F$ and $\tilde{F}$.

Let $\mathbf{F}_{\lambda}$ be a class of germs of holomorphic mappings strictly equivalent to the germ $F_{\lambda}$. In this work we restrict ourselves by studying a strict analytical classification of the germs in the class $\mathbf{F}_{\lambda}$. The germ $\mathbf{F}_{\lambda}$ is called a normal form of class $\mathbf{F}_{\lambda}$.

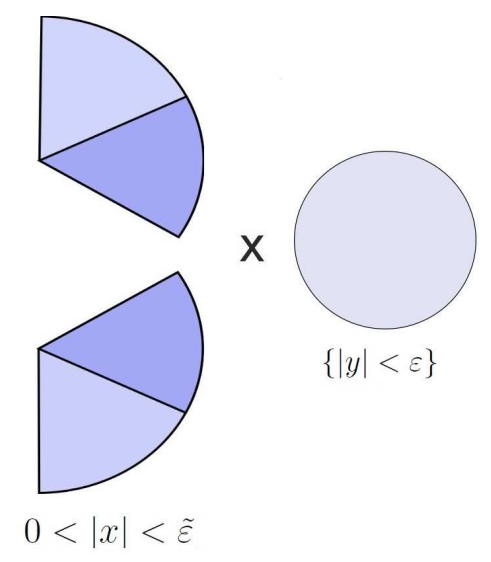

Figure 1. Sectorial domains.

The domains $\Omega^{ \pm}$of form

$$
\begin{aligned}
& \Omega^{+}=\left\{0<|x|<\tilde{\varepsilon}, \arg x \in\left(-\delta, \frac{\pi}{2}\right)\right\} \times\{|y|<\varepsilon\}, \\
& \Omega^{-}=\left\{0<|x|<\tilde{\varepsilon}, \arg x \in\left(-\frac{\pi}{2}, \delta\right)\right\} \times\{|y|<\varepsilon\} .
\end{aligned}
$$

are called an upper right or lower right sectorial domain.

As it was shown in work [19], for all $F \in \mathbf{F}_{\lambda}$ there exists the unique semi-formal normalized change of coordinates $\mathrm{H}$ adjoining the normal form $F_{\lambda}$ with the germ $F$. This change $\mathrm{H}$ is a formal series in the variable " $x$ " with coefficients holomorphic in " $y$ ".

The main result of the work is the following theorem.

Theorem 1 (On sectorial normalization in right half-neighbourhood). Let the germ $\mathrm{F}$ be in the class $\mathbf{F}_{\lambda}$, then

1. There exists a sectorial domain $\Omega^{ \pm}$such that there exists the unique holomorphic normed mapping $H^{ \pm}$adjoining normal form $F_{\lambda}$ with a representative of the germ $F$ on $\Omega^{ \pm}$;

2. Let $\mathrm{H}$ be a semi-formal normed change of coordinates normalizing $\mathrm{F}$, then $\mathrm{H}$ is an asymptotic series (as $x \rightarrow 0$ ) for a holomorphic normalizing mapping $H^{ \pm}$. 


\section{PRoOF OF THEOREM ON SECTORIAL NORMALIZATION}

3.1. Preliminary normalization. It follows from theorem on semi-formal normalization [19] that if $\mathrm{H}_{N}$ is a partial sum of normalizing semi-formal series $\mathrm{H}$, then $\mathrm{H}_{N}$ is a holomorphic change of coordinates and it maps the germ $\mathrm{F}$ into the germ $\mathrm{F}_{N}$ such that

$$
F_{N}(x, y)=F_{\lambda}(x, y)+\left(O\left(x^{N}\right), O\left(x^{N}\right)\right) \quad \text { as } \quad x \rightarrow 0 .
$$

The mapping $F_{N}$ obtained in such way is called preliminary normal form for the germ $\mathrm{F}$.

We choose arbitrary $N>6$; the parameter $N$ will be fixed later in the further calculations, see Lemmata 5-7. We seek a holomorphic change of variables $H_{N}$ adjoining normal form $F_{\lambda}$ with a preliminary normal form $F_{N}$ :

$$
F_{N} \circ H_{N}=H_{N} \circ F_{\lambda} .
$$

It is more convenient to make the analytic normalization in the coordinates

$$
(\xi, z)=B(x, y)=\left(\xi=-\frac{1}{x}, z=y\right) .
$$

In these coordinates, the preliminary normal formal $F_{N}$ in (3) reads as

$$
\begin{aligned}
& \tilde{F}_{N}(\xi, z)=F_{0}(\xi, z)+\left(\Delta_{1 N}, \Delta_{2 N}\right) \\
& \Delta_{1 N}=O\left(\xi^{-N_{1}}\right), \Delta_{2 N}=O\left(\xi^{-N_{1}}\right), \quad|\xi| \rightarrow \infty,
\end{aligned}
$$

where $F_{0} \stackrel{\text { def }}{=} B \circ F_{\lambda} \circ B^{-1}, F_{0}(\xi, z)=\left(\xi+1, e^{\lambda} z\right), N_{1}=N-2$, see [20].

A polynomial in " $x$ " transformation $\mathrm{H}_{N}$ adjoining $F_{N}$ with $F$ written in terms of the coordinates $(\xi, z)$ is denoted by $\tilde{\mathrm{H}}_{N}$.

Let the holomorphic transformation

$$
\tilde{H}_{N} \stackrel{\text { def }}{=} B \circ H_{N} \circ B^{-1}
$$

adjoining normal form $F_{0}$ with $\tilde{F}_{N}$ reads as

$$
\tilde{H}_{N}(\xi, z)=\left(\xi+h_{N}(\xi, z), z+g_{N}(\xi, z)\right) .
$$

Then the functions $h_{N}(\xi, z)$ and $g_{N}(\xi, z)$ satisfy the pair of functional equations

$$
h_{N} \circ F_{0}-h_{N}=\Delta_{1 N} \circ \tilde{H}_{N}, \quad g_{N} \circ F_{0}-e^{\lambda} g_{N}=\Delta_{2 N} \circ \tilde{H}_{N} .
$$

Assuming that $h_{N}, g_{N}, \Delta_{1 N}, \Delta_{2 N}$ are small and neglecting the terms of orders higher than one, by the functional equations we obtain so-called homological equations:

$$
\begin{aligned}
& h \circ F_{0}-h=\Delta_{1 N}, \\
& g \circ F_{0}-e^{\lambda} g=\Delta_{2 N} .
\end{aligned}
$$

We prove theorem on sectorial normalization as follows.

Step 1. Construction of a pair $(h, g)$ of solutions to homological equations in some sectorial domains.

Step 2. Construction of auxiliary operators, namely, the operator resolving homological equations $\mathcal{L}:\left(\Delta_{1 N}, \Delta_{2 N}\right) \rightarrow(h, g)$ and the substitution operator

$$
\mathcal{R}:(h, g) \rightarrow\left(\delta_{1 N}, \delta_{2 N}\right)=\left(\Delta_{1 N}(\xi+h, z+g), \Delta_{2 N}(\xi+h, z+g)\right) .
$$

Hence, the fixed point $\left(h_{N}, g_{N}\right)$ of the operator $\mathcal{H}=\mathcal{L} \circ \mathcal{R}$ is the unique solution $\tilde{H}_{N}=$ $i d+\left(h_{N}, g_{N}\right)$ of the functional equations

$$
\mathcal{H}\left[\left(h_{N}, g_{N}\right)\right]=\left(h_{N}, g_{N}\right) .
$$

The contracting property of the operator $\mathcal{H}$ will be ensured by an appropriate choice of the parameters of the sectorial domains and corresponding metric spaces. The theorem 
on contracting mappings ensures the existence and uniqueness of the normalizing sectorial mapping for $\tilde{F}_{N}$ as well as appropriate estimates for this mapping.

Step 3. The composition of a partial sum of a semi-formal normalizing change of coordinates $\mathrm{H}_{N}$ and of a holomorphic normalizing sectorial mapping $H_{N}$ is a sectorial normalizing mapping $H$. The asymptotics in Statement 2 of the theorem is implied by the uniqueness of the sectorial normalizing mapping and an arbitrary choice of $N$.

\subsection{Solutions for homological equations.}

Definition 2. A solution to homological equation in some domain $W$ is a holomorphic function $u$ satisfying this equation in $W$. The norm of the solution on $W$ is introduced in a standard way:

$$
\|u\|_{W}=\sup _{W}|u(\xi, z)| .
$$

Definition 3. We denote by $\mathbf{D}_{m}(W)$ the class of functions $d$ holomorphic in $W$ and continuous on $\bar{W}$ with a finite norm

$$
\|d\|_{W, m}=\sup _{W}\left|d(\xi, z)\left(1+|\xi|^{2}\right)^{\frac{m}{2}}\right|<+\infty, \quad m>3 .
$$

We shall employ the parameter $m$ for the brevity of writing. Its choice will be specified later while constructing the solutions to the functional equations.

Definition 4. The domain $S_{\xi}=\{\operatorname{Re} \xi<-R<-1\}$ is called the left sectorial domain in $\xi$ plane. The domain $S_{\xi}^{ \pm}$obtained as an intersection of $S_{\xi}$ and the domain $\{-\delta< \pm(\pi-\arg \xi)<$ $\left.\frac{\pi}{2}\right\}$ is called upper left, respectively, lower left sectorial domain in the plane $\xi$. The parameter of opening $\delta \in\left(\frac{\pi}{4} ; \frac{\pi}{2}\right)$ will be determined later in Proposition 3.

Definition 5. A left (upper left or lower left) sectorial domain in $(\xi, z)$-plane is a Cartesian product of $S_{\xi}$, respectively, $S_{\xi}^{ \pm}$, and the disk $\{|z|<\varepsilon\}$. We denote these domains by $S$ and $S^{ \pm}$, respectively.

Lemma 1 (On solution of first homological equation). Let $\Delta_{1 N}$ be in the class $\mathbf{D}_{m}(S)$, then there exists a holomorphic solution $h$ of the first homological equation in the left sectorial domain $S$ such that

1. $\|h\|_{S} \leqslant c_{1}\left\|\Delta_{1 N}\right\|_{S, m}$ where $c_{1}=c_{1}(m)$;

2. $h(\xi, z)=O\left(|\xi|^{-m+1}\right)$ as $|\xi| \rightarrow \infty,(\xi, z) \in S$;

3. the solution of the first homological equation in the left sectorial domain is unique in the class of holomorphic bounded functions obeying condition 2.

Lemma 2 (On solution of second homological equation). Let $\Delta_{2 N}$ be in the class $\mathbf{D}_{m}(S)$, then there exists holomorphic solution $g^{ \pm}$of the second homological equation in the domain $S$ such that

1. $\left\|g^{ \pm}\right\|_{S} \leqslant c_{2}(m, \lambda)\left\|\Delta_{2 N}\right\|_{S, m}$, where $c_{2}=c_{2}(m, \lambda)$;

2. $g^{ \pm}(\xi, z)=O\left(|\xi|^{-m+3}\right)$ as $|\xi| \rightarrow+\infty,(\xi, z) \in S^{ \pm}$;

3. Let the parameter $\delta \in\left(\frac{\pi}{4} ; \frac{\pi}{2}\right)$ be such that $\tan \delta>\frac{\lambda}{2 \pi}$, then the solution of the second homological equation in the upper left (lower left) sectorial domain is unique in the class of holomorphic bounded functions obeying condition 2 respectively in $S^{ \pm}$.

Proof of Lemma 1. The solution of the first homological equation can be represented as

$$
h(\xi, z)=\sum_{n=1}^{+\infty} \Delta_{1 N}\left(\xi-n, e^{-\lambda n} z\right) .
$$

It is easy to confirm that all functions in the right hand side are well-defined, the series converges uniformly in $S$ and its sum is a holomorphic function on $S$ and this is the function $h$ indeed solves the first homological equation in $S$. 
Let us show the uniqueness. Assume that $h_{1}$ and $h_{2}$ are solutions to the first homological equation in the domain $S$ obeying condition 2 . We let $r=h_{1}-h_{2}$. Then in the domain $S$ the function $r$ satisfies:

$$
r\left(\xi+1, e^{\lambda} z\right)-r(\xi, z)=0
$$

and $r$ can be continued on $\mathbb{C}^{2}$ as follows. Let $(\xi, z)$ be a point in $\mathbb{C}^{2}$ outside $S$. Then there exist $n_{1}=[\operatorname{Re} \xi+R]+1$ and $n_{2}=\left[\log _{e^{\lambda}} \frac{1}{\varepsilon}\right]+1$ such that for $n=\max \left\{n_{1} ; n_{2}\right\}$, the point $\left(\xi_{0}, z_{0}\right)=\left(\xi-n, e^{-\lambda n} z\right)$ is contained in the domain $S$ and we can define the value of the function $r$ at the point $(\xi, z)$ via the value at the point $\left(\xi_{0}, z_{0}\right)$, namely,

$$
r(\xi, z)=r\left(\xi-n, e^{-\lambda n} z\right)=r\left(\xi_{0}, z_{0}\right)
$$

Since $r$ is holomorphic and bounded in $S$ and satisfies $(11)$, its continuation on $\mathbb{C}^{2}$ is holomorphic and bounded on $\mathbb{C}^{2}$. By Liouville theorem [23] we then get that $r=$ const. In view of condition 2 we hence get $r \equiv 0$. The proof is complete.

Proof of Lemma 2. We consider the restriction of the second homological equation on

$$
S_{0} \stackrel{\text { def }}{=}\{(\xi, z) \in S: z=0\} .
$$

We observe that it follows from the definition of the left sectorial domain that $S_{0}=S_{\xi}$. We let

$$
g_{0}(\xi) \stackrel{\text { def }}{=} g(\xi, 0), \quad \Delta_{0}(\xi) \stackrel{\text { def }}{=} \Delta_{2 N}(\xi, 0) .
$$

Then the function $g_{0}$ can be found as a solution to the equation

$$
g_{0}(\xi+1)-e^{\lambda} g_{0}(\xi)=\Delta_{0}(\xi), \quad \xi \in S_{\xi} .
$$

We multiply equation 12 by $e^{-\lambda(\xi+1+R)}$ and we let

$$
d(\xi) \stackrel{\text { def }}{=} e^{-\lambda(\xi+1+R)} \Delta_{0}(\xi) .
$$

Then equation $(12)$ becomes

$$
u(\xi+1)-u(\xi)=d(\xi), \quad \xi \in S_{\xi} .
$$

The solution of this simplest functional equation in a curved strip like domain was considered in work [24]. Following Theorem 1 in this work, in $S_{\xi}$ we define a strip $\Pi$ of width $\frac{3}{2}$ as follows: the vertical straight line $L_{+} \stackrel{\text { def }}{=} \partial S_{\xi}=\{\operatorname{Re} \xi=-R\}$ is the right boundary of the strip and the vertical straight line $L_{-}=L_{+}-\frac{3}{2}$ is the left boundary and at that, $\partial \Pi=L_{+}-L_{-}$.

Since $\Delta_{2 N}$ is in the class $\mathbf{D}_{m}(S)$, the function $d$ in the right hand in equation (14) belongs to the class $\mathbf{D}_{m}(\Pi)$. Then Theorem 1 in [24] implies the following lemma.

Lemma 3 (Main lemma). The function

$$
u(\xi)=\frac{1}{2 \pi i} \sum_{n=0}^{+\infty}\left(\int_{L_{-}} \frac{d(t) d t}{t-\xi-n}+\int_{L_{+}} \frac{d(t) d t}{t-\xi+n+1}\right)
$$

solves equation (14) with a right hand side $d$ from the class $\mathbf{D}_{m}(\Pi)$ in the strip $\Pi$ and

1. There exists $c(m)$ such that $\|u\|_{\Pi} \leqslant c(m)\|d\|_{\Pi, m}$;

2. Let $M_{0}^{R}=\frac{1}{2} \int_{L_{-}} d(t) d t$, then

$$
\sup _{\Pi}\left|u(\xi) \mp M_{0}^{R}\right| \leqslant \frac{C(m)\|d\|_{\Pi, m}}{|\xi|^{m-3}}, \quad \xi \in \Pi, \quad \pm \operatorname{Im} \xi>|\operatorname{Re} \xi| ;
$$

3. Solution of equation (14) is unique in the class of holomorphic bounded functions satisfying condition 2 in the strip $\Pi$. 
We let

$$
M_{0}(a)=\frac{1}{2} \int_{\operatorname{Re} t=a} \Delta_{0}(t) e^{-\lambda(t+1)} d t .
$$

We note that as $a=-R-\frac{3}{2}$, we have $M_{0}=M_{0}^{R} e^{\lambda R}$.

Proposition 1. The choice of value $M_{0}$ is independent on $a<-R$.

Proof. The statement can be obtained from the Cauchy theorem in the standard way since the condition $\Delta_{2 N} \in \mathbf{D}_{m}(S)$ ensures uniform estimates for the integrand as $\operatorname{Im} \xi \rightarrow \infty, \xi \in S$.

Let $u$ be a solution of equation (14) with the right hand side $d \in \mathbf{D}_{m}(\Pi)$ defined in (13) in Lemma 3 .

We let

$$
g_{0}(\xi) \stackrel{\text { def }}{=} u(\xi) e^{\lambda(\xi+R)}, \quad \xi \in \Pi,
$$

and, in view of $(12)$, we continue $g_{0}$ from strip $\Pi$ to the half-plane $\tilde{S}_{l}$ by the formula:

$$
g_{0}(\xi) \stackrel{\text { def }}{=} e^{-\lambda k} g_{0}(\xi+k)-\sum_{n=0}^{k-1} e^{-\lambda(n+1)} \Delta_{0}(\xi+n), \quad k=[-R-\operatorname{Re} \xi], \quad \xi \in S_{\xi} \backslash \Pi .
$$

Proposition 2. The function $g_{0}$ defined by formulae (15) and (16) are holomorphic bounded solution of equation (12) on $S_{\xi}$ and

1. There exists $c=c(m, \lambda)$ such that $\left\|g_{0}\right\|_{\tilde{S}_{l}} \leqslant c\left\|\Delta_{0}\right\|_{S_{\xi}, m}$;

2. $\left|g_{0}(\xi) \mp M_{0} e^{\lambda \xi}\right|=O\left(|\xi|^{-m+3}\right), \xi \in \tilde{S}_{l}^{ \pm},|\xi| \rightarrow \infty$.

Remark 1. Since generally speaking $M_{0}$ is non-zero, the estimate from Statement 2 in Proposition 2 is not extended on $S_{\xi}=S_{\xi}^{+} \cup S_{\xi}^{-}$.

Proof of Proposition 2. Since the width of the strip $\Pi$ is greater than 1, for each point $\xi$ to the left of the strip $(\xi+k), k=[-R-\operatorname{Re} \xi]$ is located in the strip $\Pi$. Hence, the solution $g_{0}$ defined by formula 16 is well-defined on $S_{\xi}$ and the following facts hold.

$\diamond$ The function $g_{0}$ satisfies equation 12 that can be confirmed by straightforward calculations.

$\diamond$ The function $g_{0}$ is holomorphic as a finite sum of holomorphic functions on each strip $\{\xi: \operatorname{Re} \xi \in(-A-1 ;-A), A \in \mathbb{N}\}$. We note that the function $g_{0}$ defined by formulae (15) and (16) satisfies equation $(12)$ and hence, all points in the straight line $\left\{\xi \in S_{\xi}: \operatorname{Re} \xi \in \mathbb{Z}_{-}\right\}$are removable and therefore, $g_{0}$ is holomorphic on $S_{\xi}$.

$\diamond$ Lemma 3 implies the following estimate for $g_{0}$ on the strip $\Pi$ :

$$
\left\|g_{0}\right\|_{\Pi}=\left\|u(\xi) e^{\lambda(\xi+R)}\right\|_{\Pi} \leqslant c(m)\left\|\Delta_{0}(\xi) e^{-\lambda(\xi+R+1)}\right\|_{\Pi, m} \leqslant c(m)\left\|\Delta_{0}\right\|_{\Pi, m} .
$$

Let us estimate the norm of $g_{0}$ continued on $S_{\xi} \backslash \Pi$; we recall that $\lambda \in \mathbb{R}_{+}$, that is, $e^{-\lambda}<1$ :

$$
\begin{aligned}
\left\|g_{0}\right\|_{S_{\xi} \backslash \Pi} & \leqslant e^{-\lambda(k+1)}\left\|g_{0}\right\|_{\Pi}+\frac{e^{-\lambda}-e^{-\lambda(k+1)}}{1-e^{-\lambda}}\left\|\Delta_{0}\right\|_{S_{\xi}, m} \\
& \leqslant c(m)\left\|\Delta_{0}\right\|_{\Pi, m}+\frac{1}{e^{\lambda}-1}\left\|\Delta_{0}\right\|_{S_{\xi}, m} \\
& \leqslant\left(c(m)+\frac{1}{e^{\lambda}-1}\right)\left\|\Delta_{0}\right\|_{S_{\xi}, m}=c(m, \lambda)\left\|\Delta_{0}\right\|_{S_{\xi}, m} .
\end{aligned}
$$

Hence,

$$
\left\|g_{0}\right\|_{S_{\xi}} \leqslant c(m, \lambda)\left\|\Delta_{0}\right\|_{S_{\xi}, m}
$$

This proves the first statement of the proposition. 
$\diamond$ Statement 2 of Lemma 3 provides an asymptotic estimate for the solution in the strip $\Pi$; we recall that $g_{0}(\xi)=u(\xi) e^{\lambda(\xi+R)}, \Delta_{0}(\xi)=d(\xi) e^{\lambda(\xi+R+1)}$ and $\left.M_{0} e^{\lambda \xi}=M_{0}^{R} e^{\lambda(\xi+R)}\right)$ :

$$
\left|g_{0}(\xi) \mp M_{0} e^{\lambda \xi}\right| \leqslant \frac{C(m)\left\|\Delta_{0}\right\|_{\Pi, m}}{|\xi|^{m-3}}, \quad \pm \operatorname{Im} \xi>|\operatorname{Re} \xi|, \quad \xi \in \Pi .
$$

We continue the function $g_{0}$ from the strip $\Pi$ on $S_{\xi}$ by formula (16) keeping for the continuation the same notation. Then, if $|\operatorname{Im} \xi|>|\operatorname{Re} \xi|$, then

$$
\begin{aligned}
\left|g_{0}(\xi) \mp M_{0} e^{\lambda \xi}\right| & =\left|e^{-\lambda k} g_{0}(\xi+k) \mp M_{0} e^{\lambda(\xi+k)} e^{-\lambda k}-\sum_{n=0}^{k-1} e^{-\lambda(n+1)} \Delta_{0}(\xi+n)\right| \\
& \leqslant\left|e^{-\lambda k}\left(g_{0}(\xi+k) \mp M_{0} e^{\lambda(\xi+k)}\right)\right|+\left|\sum_{n=0}^{k-1} e^{-\lambda(n+1)} \Delta_{0}(\xi+n)\right|
\end{aligned}
$$

for each point $\xi$ in the domain $S_{\xi}$. Since the point $\xi+k$ is inside the strip $\Pi$, the first term satisfies inequality (17). Estimating the second term, we finally get:

$$
\left|g_{0}(\xi) \mp M_{0} e^{\lambda \xi}\right| \leqslant \frac{C(m)\left\|\Delta_{0}\right\|_{\Pi, m}}{|\xi+k|^{m-3} e^{\lambda k}}+\sum_{n=0}^{k-1} \frac{\left\|\Delta_{0}\right\|_{S_{\xi}, m}}{|\operatorname{Im} \xi|^{m} e^{\lambda n}}, \quad \pm \operatorname{Im} \xi>|\operatorname{Re} \xi|, \quad \xi \in S_{\xi} .
$$

We note that in the considered case, for sufficiently great imaginary part of $\xi$ we have:

$$
|\xi+k|>|\operatorname{Im} \xi|>\frac{|\xi|}{2} .
$$

Hence,

$$
\left|g_{0}(\xi) \mp M_{0} e^{\lambda \xi}\right| \leqslant \frac{\hat{C}(m, \lambda)\left\|\Delta_{0}\right\|_{S_{\xi}, m}}{|\xi|^{m-3}}, \quad \pm \operatorname{Im} \xi>|\operatorname{Re} \xi|, \quad \xi \in S_{\xi} .
$$

If $\xi$ is located inside the sector $|\operatorname{Im} \xi| \leqslant \tan \delta|\operatorname{Re} \xi| \operatorname{Re} \xi<-R$, then it is sufficient to prove the asymptotics of form $O\left(|\operatorname{Re} \xi|^{-m+3}\right)$ since as $|\operatorname{Im} \xi| \leqslant \tan \delta|\operatorname{Re} \xi|$, we have $|\xi| \leqslant(1+\tan \delta)|\operatorname{Re} \xi|$, that is, $|\operatorname{Re} \xi| \geqslant \frac{|\xi|}{1+\tan \delta}$.

We construct a solution $g_{0}$ to equation $(12)$ in $S_{\xi}$ by the above described scheme: let $\xi \in S_{\xi}$, then there exists a natural number $k=[-\operatorname{Re} \xi-R]$ such that $\xi+k$ is located in the strip $\Pi$. At that, since $\xi+k$ is located in the strip $\Pi$, then $e^{-\lambda(\xi+k)}$ and $g_{0}(\xi+k)$ are bounded thanks to Lemma 3. Hence,

$$
\begin{aligned}
\left|g_{0}(\xi) \mp M_{0} e^{\lambda \xi}\right| & =\left|e^{-\lambda k}\left(g_{0}(\xi+k) \mp M_{0} e^{\lambda(\xi+k)}\right)-\sum_{n=0}^{k-1} e^{-\lambda(n+1)} \Delta_{0}(\xi+n)\right| \\
& \leqslant \frac{c\left\|\Delta_{0}\right\|_{\Pi, m}}{e^{\lambda k}}+\sum_{n=0}^{k-1} \frac{2^{m}\left\|\Delta_{0}\right\|_{S_{\xi}, m} e^{-\lambda(n+1)}}{(1+|\operatorname{Re} \xi+n|)^{m}} \\
& \leqslant \frac{c\left\|\Delta_{0}\right\|_{\Pi, m} e^{\lambda R}}{e^{\lambda|\operatorname{Re} \xi|}}+\sum_{n=0}^{k-1} \frac{2^{m}\left\|\Delta_{0}\right\|_{S_{\xi}, m} e^{-\lambda(n+1)}}{(1+|\operatorname{Re} \xi+n|)^{m}} .
\end{aligned}
$$

We have

as

$$
\frac{\partial}{\partial n}\left(e^{-\lambda(n+1)}(1+|\operatorname{Re} \xi|-n \mid)^{-m}\right)=0
$$

$$
n_{0}=\frac{-m}{\lambda}+1+|\operatorname{Re} \xi|
$$

For sufficiently large $|\operatorname{Re} \xi|$ we also have $n_{0} \in \mathbb{R}_{+}, k \leqslant|\operatorname{Re} \xi|$ and therefore, $\sum_{n=0}^{k-1} e^{-\lambda(n+1)}(1+|\operatorname{Re} \xi+n|)^{-m}=\sum_{n=0}^{k-1} e^{-\lambda(n+1)}(1+|\operatorname{Re} \xi|-n)^{-m} \leqslant|\operatorname{Re} \xi| \cdot\left(\frac{\lambda}{m}\right)^{m} e^{m-2 \lambda} e^{-|\operatorname{Re} \xi|}$. 
Then for some constant $\tilde{C}(m, \lambda, \delta)$ the inequalities hold:

$$
\left|g_{0}(\xi) \mp M_{0} e^{\lambda \xi}\right| \leqslant \frac{\tilde{C}(m, \lambda, \delta)\left\|\Delta_{0}\right\|_{S_{\xi}, m}}{|\xi|^{m}}, \quad|\operatorname{Im} \xi|<\tan \delta|\operatorname{Re} \xi|, \quad \xi \in S_{\xi} .
$$

Inequalities (18), (19) and the condition $\delta \in\left(\frac{\pi}{4} ; \frac{\pi}{2}\right)$ yield the second statement of the proposition. The proof is complete.

Proposition 3. For each $\delta \in\left(\frac{\pi}{4} ; \frac{\pi}{2}\right)$ such that $\tan \delta>\frac{\lambda}{2 \pi}$, a holomorphic bounded on $S_{\xi}$ solution to equation (12) satisfying Statement 2 of Proposition 2 is unique.

Proof. Let $g_{1}^{ \pm}$and $g_{2}^{ \pm}$are holomorphic bounded solutions of the equation:

$$
g_{0}(\xi+1)-e^{\lambda} g_{0}(\xi)=\Delta_{0}(\xi), \quad \xi \in S_{\xi},
$$

and satisfy Statement 2 of Proposition 2 on $S_{\xi}^{ \pm}$. We let

$$
g^{ \pm}(\xi) \stackrel{\text { def }}{=} g_{1}^{ \pm}(\xi)-g_{2}^{ \pm}(\xi)
$$

Then

$$
g^{ \pm}(\xi+1)-e^{\lambda} g^{ \pm}(\xi)=0, \xi \in S_{\xi}^{ \pm}
$$

We denote $u^{ \pm}(\xi) \stackrel{\text { def }}{=} g^{ \pm}(\xi) e^{-\lambda \xi}$. Then the function $u^{ \pm}$is holomorphic on $S_{\xi}$ and is 1-periodic.

We consider the domain $S_{\xi}^{+}$. For each $\xi$ in $S_{\xi}^{+}$there exists a natural $n$ such that $\tilde{\xi}=\xi+n$ is located inside a broken strip:

$$
\Pi^{+}=\left\{\begin{array}{lll}
-R-1 \leqslant \operatorname{Re} \xi \leqslant-R, & \text { as } & \operatorname{Im} \xi>-R \tan \delta \\
c \tan \delta \operatorname{Im} \xi-1 \leqslant \operatorname{Re} \xi \leqslant c \tan \delta \operatorname{Im} \xi, & \text { as } & \operatorname{Im} \xi \leqslant-R \tan \delta
\end{array}\right.
$$

By 1-periodicity we have $u^{+}(\tilde{\xi})=u^{+}(\xi)$. We are going to show the boundedness of the function

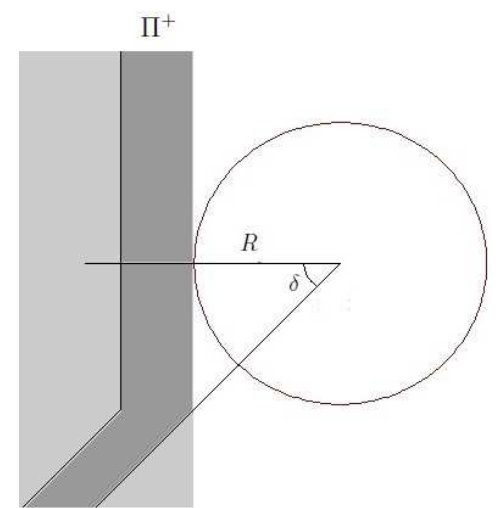

FiguRE 2. Strip $\Pi^{+}$.

$u^{+}$on the strip $\Pi^{+}$. We let

$$
J: \xi \mapsto t=e^{2 \pi i \xi}
$$

We note that

$$
\begin{aligned}
& J\left(\Pi^{+} \cap\{\operatorname{Im} \xi>-R \tan \delta\}\right)=\left\{0<|t|<e^{2 \pi R}\right\} \\
& J\left(\Pi^{+} \cap\{\operatorname{Im} \xi \leqslant-R \tan \delta\}\right)=\left\{|t| \geqslant e^{2 \pi R}\right\} .
\end{aligned}
$$

In coordinates $(20)$, the function $u^{+}$reads as

$$
\tilde{u}^{+}(t) \stackrel{\text { def }}{=} J^{-1} \circ u^{+} \circ J=t^{\frac{-\lambda}{2 \pi i}} g^{+}\left(\frac{1}{2 \pi i} \ln t\right) .
$$

By 1-periodicity of the function $u^{+}$, the function $\tilde{u}^{+}$is well-defined and is holomorphic on $\mathbb{C}_{*}=J\left(S_{\xi}\right)$. Moreover,

$$
|t|=\left|e^{2 \pi i \xi}\right|=e^{-2 \pi \operatorname{Im} \xi} .
$$


In the image $\left\{0<|t|<e^{2 \pi R}\right\}$ of the upper part of the strip we have:

$$
\begin{aligned}
& \left|t^{\frac{-\lambda}{2 \pi i}}\right|=e^{-\lambda \operatorname{Re} \xi}=e^{-\lambda \operatorname{Re} \tilde{\xi}} \leqslant e^{\lambda(R+1)}, \\
& \left|\tilde{u}^{+}(t)\right| \leqslant\left|g^{+}\left(\frac{1}{2 \pi i} \ln t\right)\right| e^{\lambda(R+1)} \leqslant \text { Const } \quad \text { as } \quad\left\{0<|t|<e^{2 \pi R}\right\} .
\end{aligned}
$$

Then in the image of the lower part of the strip, that is, as $\left\{|t| \geqslant e^{2 \pi R}\right\}$, we have:

$$
\begin{aligned}
& \left|t^{\frac{-\lambda}{2 \pi i}}\right|=e^{-\lambda \operatorname{Re} \xi}=e^{-\lambda \operatorname{Re} \tilde{\xi}} \leqslant e^{-\frac{\lambda \operatorname{Im} \tilde{\xi}}{\tan \delta}} e^{\lambda}=e^{-\frac{\lambda \operatorname{Im} \xi}{\tan \delta}} e^{\lambda}=e^{\lambda}|t| \frac{\lambda}{2 \pi \tan \delta}, \\
& \left|\tilde{u}^{+}(t)\right| \leqslant|t|^{\frac{\lambda}{2 \pi \tan \delta}}\left|g^{+}\left(\frac{1}{2 \pi i} \ln t\right)\right| \leqslant \text { Const }|t| \frac{\lambda}{2 \pi \tan \delta} \quad \text { as } \quad|t| \geqslant e^{2 \pi R} .
\end{aligned}
$$

Since $\tan \delta>\frac{\lambda}{2 \pi}$, by the Cauchy estimates for the coefficients of the Taylor series [23] we infer that $t=\infty$ is a removable singular point of the function $\tilde{u}^{+}$and hence, $\tilde{u}^{+}$is bounded as $|t| \geqslant e^{2 \pi R}$. In view of $(21)$, the function $\tilde{u}^{+}$is also bounded on $\mathbb{C}_{*}$.

Since $\tilde{u}^{+}$is holomorphic on $\mathbb{C}_{*}$, by the Riemann theorem on a removable singularity 23 ] we conclude that $\tilde{u}^{+}$is holomorphic and bounded on $\mathbb{C}$. Then Liouville theorem implies that $\tilde{u}^{+}=$const . Then $g^{+}(\xi)=$ const $\cdot e^{\lambda \xi}$ by the asymptotic properties of the function $g^{+}$, see Statement 2 in Proposition 2. Since the rays $\{\xi: \operatorname{Re} \xi=$ const $\leqslant-R, \operatorname{Im} \xi \geqslant 0\}$ belong to the domain $S_{\xi}^{+}$, we infer that $g^{+} \equiv 0$.

In the same way we consider the domain $S_{\xi}^{-}=\{\operatorname{Re} \xi \leqslant-R, \operatorname{Im} \xi \leqslant-\tan \delta \operatorname{Re} \xi\}$. For each $\xi \in S_{\xi}^{-}$there exists a natural $n$ such that $\tilde{\xi}=\xi+n$ is located inside the broken strip $\Pi^{-}$:

$$
\Pi^{-}=\left\{\begin{array}{lll}
-R-1 \leqslant \operatorname{Re} \xi \leqslant-R, & \text { if } & \operatorname{Im} \xi<R \tan \delta, \\
-c \tan \delta \operatorname{Im} \xi-1 \leqslant \operatorname{Re} \xi \leqslant-c \tan \delta \operatorname{Im} \xi, & \text { if } \quad \operatorname{Im} \xi \geqslant R \tan \delta .
\end{array}\right.
$$

We apply mapping $(20)\left(J: \xi \mapsto\left(t=e^{2 \pi i \xi}\right)\right)$ :

$$
\begin{aligned}
& J\left(\Pi^{-} \cap\{\operatorname{Im} \xi<R \tan \delta\}\right)=\left\{|t|>e^{-R \tan \delta}\right\}, \\
& J\left(\Pi^{-} \cap\{\operatorname{Im} \xi \geqslant R \tan \delta\}\right)=\left\{0<|t| \leqslant e^{-R \tan \delta}\right\}, \\
& \tilde{u}^{-}(t) \stackrel{\text { def }}{=} J^{-1} \circ u^{+} \circ J=t^{\frac{-\lambda}{2 \pi i}} g^{-}\left(\frac{1}{2 \pi i} \ln t\right) .
\end{aligned}
$$

Similar to the above arguing, by 1-periodicity of the function $u^{-}$, the function $\tilde{u}^{-}$is well-defined and holomorphic on $\mathbb{C}_{*}$. Moreover, by the boundedness of $g^{-}$we have:

$$
\left|\tilde{u}^{-}(t)\right| \leqslant \text { Const }\left\{\begin{array}{lll}
e^{\lambda(R+1)} & \text { if } & |t|>e^{-R \tan \delta}, \\
e^{\lambda}|t| \frac{-\lambda}{2 \pi \tan \delta} & \text { if } & 0<|t| \leqslant e^{-R \tan \delta}
\end{array}\right.
$$

It follows from the Cauchy estimates for the coefficients of the Laurent series [23] that $t=0$ is a removable singular point $\tilde{u}^{-}$and this is why $\tilde{u}^{-}$can be continued to a holomorphic and bounded on $\mathbb{C}$ function. This is why $\tilde{u}^{-}=$const on $\mathbb{C}$. As above, this imples $g^{-} \equiv 0$. This completes the proof of Lemma 2 .

We let $g_{0}^{ \pm}(\xi) \stackrel{\text { def }}{=} g_{0}(\xi) \mp M_{0} e^{\lambda \xi}$. We have constructed the solutions to the reduced second homological equation (12) in the domain $S_{\xi}$. We deduct equation (12) from the second homological equation. We obtain one more equation:

$$
r\left(\xi+1, e^{\lambda} z\right)-e^{\lambda} r(\xi, z)=\rho_{N}(\xi, z), \quad(\xi, z) \in S,
$$

where

$$
\rho_{N}(\xi, z) \stackrel{\text { def }}{=} \Delta_{2 N}(\xi, z)-\Delta_{0}(\xi), \quad r(\xi, z) \stackrel{\text { def }}{=} g(\xi, z)-g_{0}(\xi)
$$


It follows from the Schwarz lemma 23 that $\rho_{N}(\xi, z)=z \tilde{\Delta}_{N}(\xi, z)$, where $\tilde{\Delta}_{N}$ is in the class $\mathbf{D}_{m}(S)$. We seek a solution to equation (22) in the left sectorial domain $S$ as $r(\xi, z)=z \tilde{g}(\xi, z)$, where $\tilde{g}(\xi, z)$ satisfies the equation

$$
\tilde{g}\left(\xi+1, e^{\lambda} z\right)-\tilde{g}(\xi, z)=e^{-\lambda} \tilde{\Delta}_{N}(\xi, z), \quad(\xi, z) \in S .
$$

Since the function $e^{-\lambda} \tilde{\Delta}_{N}$ is in the class $\mathbf{D}_{m}(S)$, the following proposition is in fact a reformulation of Lemma 1.

Proposition 4. The function $\tilde{g}(\xi, z)=\sum_{n=1}^{+\infty} e^{-\lambda} \tilde{\Delta}_{N}\left(\xi-n, e^{-\lambda n} z\right)$ is a solution to equation (23) and $\tilde{g}$ is holomorphic in $S$ and

1. For some constant $c=c(m)$ an inequality holds: $\|\tilde{g}\|_{S} \leqslant c\left\|\tilde{\Delta}_{N}\right\|_{S, m}$;

2. $\tilde{g}(\xi, z)=O\left(|\xi|^{-m+1}\right)$ as $|\xi| \rightarrow \infty,(\xi, z) \in S$;

3. The solution of equation (23) is unique in $S$ in the class of holomorphic bounded functions obeying condition 2.

Propositions 2-4 provide a needed solution to the second homological equation in the domain $S$ of form:

$$
g^{ \pm}(\xi, z)=g_{0}^{ \pm}(\xi)+z \tilde{g}(\xi, z), \quad(\xi, z) \in S,
$$

where $g_{0}^{ \pm}$comes from Propositions 2,3, and $\tilde{g}$ is from Proposition 4.

We note that since for each pair $(\xi, z)$ in $S$ we have $\operatorname{Re} \xi<-R$, there exists $C(\lambda)$ such that

$$
\left\|M_{0} e^{\lambda \xi}\right\|_{S}=\sup _{S}\left|\frac{1}{2} \int_{L} \Delta_{0}(t) e^{-\lambda(t-\xi)} d t\right| \leqslant C(\lambda)\left\|\Delta_{0}\right\|_{S, m},
$$

where $L=\left\{\operatorname{Re} t=-R-\frac{3}{2}\right\}$. Hence, we have proved Lemma 2 ; other estimates follow corresponding estimates in Propositions 2-4.

3.3. Solutions of functional equations. This section is devoted to the second step in the proof of the theorem on sectorial normalization. Here we construct auxiliary operators $\mathcal{L}$ and $\mathcal{R}$ and we also prove the contracting property of the operator $\mathcal{H}=\mathcal{L} \circ \mathcal{R}$ under an appropriate choice of the parameters of the sectorial domains.

For a vector function $d=\left(d_{1}, d_{2}\right)$ holomorphic in the left sectorial domain $S$ we let:

$$
\|d\|_{S, m} \stackrel{\text { def }}{=}\left\|d_{1}\right\|_{S, m}+\left\|d_{2}\right\|_{S, m} .
$$

We denote by $\mathbf{B}_{m}(S)$ a normed space consisting of the vector functions $d=\left(d_{1}, d_{2}\right)$ holomorphic on $S$ with a finite norm $\|d\|_{S, m}<\infty$ defined by (24).

For a vector function $f=(h, g)$ holomorphic on the left sectorial domain $S$ we let

$$
\|f\|_{S} \stackrel{\text { def }}{=}\|h\|_{S}+\|g\|_{S}
$$

We denote by $\mathbf{A}(S)$ the space of vector functions $f=(h, g)$ holomorphic on $S$ with norm (25). We observe that the spaces $\mathbf{B}_{m}(S)$ and $\mathbf{A}(S)$ are Banach.

Resolving operator for homological equations $\mathcal{L}^{ \pm}$. We denote by $\mathcal{L}^{ \pm}$the operator resolving homological equations (6) and (7) with the right hand side $d$ in the class $\mathbf{B}_{m}(S)$, that is, the operator mapping vector functions $d$ in the class $\mathbf{B}_{m}(S)$ into the vector function $f^{ \pm}$in the class $\mathbf{A}(S))$ :

$$
f^{ \pm}=\mathcal{L}^{ \pm}[d]
$$

and acting accroding Lemmata 1 and 2. Then the following lemma follows immediately Lemmata 1 and 2 . 
Lemma 4. For each $m \in(3 ; N-2)$ the operator $\mathcal{L}^{ \pm}$is well-defined as acting from $\mathbf{B}_{m}(S)$ into $\mathbf{A}(S)$ and for some $\mathfrak{C}(m, \lambda)$ we have

$$
\left\|\mathcal{L}^{ \pm}[d]\right\|_{S} \leqslant \mathfrak{C}(m, \lambda)\|d\|_{S, m}
$$

for all $d \in \mathbf{B}_{m}(S)$.

Substitution operator $\mathcal{R}$. Let $\delta \in\left(\frac{\pi}{4} ; \frac{\pi}{2}\right)$ be such that $\tan \delta>\frac{\lambda}{2 \pi}$. Hereinafter the vector function $\Delta_{N}=\left(\Delta_{1 N}, \Delta_{2 N}\right)$ is assumed to be fixed.

We choose the parameters $R_{0}>1, \varepsilon_{0}>0$ and find $S_{R_{0} \varepsilon_{0}}$ such that $\Delta_{N}$ is holomorphic on $S_{R_{0} \varepsilon_{0}}$. Then it follows from asymptotics (4) that $\Delta_{N}$ is in the class $\mathbf{B}_{N-2}\left(S_{R_{0} \varepsilon_{0}}\right)$.

We choose a positive number $\varepsilon_{1}<\frac{\varepsilon_{0}}{2}$ and $R_{1}>\max \left\{R_{0}+1 ; \frac{1}{\varepsilon_{0}}\right\}$, then for each $\xi \in\{\operatorname{Re} \xi \leqslant$ $\left.-R_{1}\right\}$ the Cartesian product of the unit disk centered at the point $\xi$ and of the circle $|z|<\varepsilon_{0}$ is entirely contained in $S_{R_{0} \varepsilon_{0}}$. We choose $R>R_{1}, \varepsilon<\varepsilon_{1}$ and construct a sectorial domain $S_{R \varepsilon}$.

Let $\omega=\min \left\{\frac{1}{2} ; \frac{\varepsilon_{0}}{2}\right\}$. We denote by

$$
\mathbf{M}_{\omega}\left(S_{R \varepsilon}\right)=\left\{f \in \mathbf{A}\left(S_{R \varepsilon}\right):\|f\|_{S_{R \varepsilon}} \leqslant \omega\right\}
$$

a ball of radius $\omega$ centered at zero in the metric space $\mathbf{A}\left(S_{R \varepsilon}\right)$ with metric induced by norm (25).

By $\mathcal{R}$ we denote an operator acting by the rule $\mathcal{R}[(h, g)]=\left(d_{1}, d_{2}\right)$, where

$$
d_{1}(\xi, z)=\Delta_{1 N}(\xi+h(\xi, z), z+g(\xi, z)), \quad d_{2}(\xi, z)=\Delta_{2 N}(\xi+h(\xi, z), z+g(\xi, z)) .
$$

Lemma 5. For all $m \in(3 ; N-2)$ the operator $\mathcal{R}$ is well-defined and acts $\mathbf{M}_{\omega}\left(S_{R \varepsilon}\right)$ into $\mathbf{B}_{m}\left(S_{R \varepsilon}\right)$ and

1. $\|\mathcal{R}[f]\|_{S_{R \varepsilon}, m} \leqslant c_{1}$ for all $f \in \mathbf{M}_{\omega}\left(S_{R \varepsilon}\right)$ with some $c_{1}=c_{1}\left(m, R, \varepsilon_{0}\right)$;

2. The operator $\mathcal{R}$ is Lipschitz with a constant $c_{2}=c_{2}\left(m, R, \varepsilon_{0}\right)$.

A detailed proof of this lemma was given in work [20].

Proposition 5. For each $m \in(3 ; N-3)$ and for some $c=c(N)$ the inequality holds:

$$
\mathfrak{c}(R, m)=\max \left\{c_{1}, c_{2}\right\} \leqslant c R^{-N+3+m}\left\|\Delta_{N}\right\|_{S_{R_{0} \varepsilon_{0}},(N-2)}
$$

Proof. The statement follows the estimate for $m$-norm of the operator $\mathcal{R}$ via $(N-2)$-norm.

Contracting property of the composition of operators $\mathcal{H}^{ \pm} \stackrel{\text { def }}{=} \mathcal{L}^{ \pm} \circ \mathcal{R}$. We first specify the choice of sectorial domains.

We choose some $\varepsilon_{0}>0, R_{0}>1,3<m<N-3$ and construct $S_{R_{0} \varepsilon_{0}}$. Then we calculate $\left\|\Delta_{N}\right\|_{S_{R_{0} \varepsilon_{0}},(N-2)}$ and $\mathfrak{C}(m, \lambda)$ by Lemma 4 . We let

$$
\omega=\min \left\{\frac{1}{2} ; \frac{\varepsilon_{0}}{2}\right\}, \quad 0<\varepsilon_{1}<\frac{\varepsilon_{0}}{2} .
$$

Let $c(N)$ be from Proposition 5. We denote

$$
R_{1}=\max \left\{R_{0}+1 ; \frac{1}{\varepsilon_{0}} ;\left(c(N) \omega^{-1} \mathfrak{C}(m, \lambda)\left\|\Delta_{N}\right\|_{S_{R_{0} \varepsilon_{0}},(N-2)}\right)^{\frac{1}{N-m-3}}\right\} .
$$

We finally choose $R \geqslant R_{1}$ and a positive $\varepsilon \leqslant \varepsilon_{1}$. Then we construct $\mathbf{A}\left(S_{R \varepsilon}\right), \mathbf{B}_{m}\left(S_{R \varepsilon}\right)$ and $\mathbf{M}_{\omega}\left(S_{R \varepsilon}\right)$.

Lemma 6. Under the above choice of the parameters, the operator $\mathcal{H}^{ \pm}$acts from $\mathbf{M}_{\omega}\left(S_{R \varepsilon}\right)$ into $\mathbf{M}_{\omega}\left(S_{R \varepsilon}\right)$ and is contracting. 
Proof. It follows from Lemma 5 that the operator $\mathcal{R}$ acts from $\mathbf{M}_{\omega}\left(S_{R \varepsilon}\right)$ into $\mathbf{B}_{m}\left(S_{R \varepsilon}\right)$ with the constant $c_{1}$ (Statement 1) and is Lipschitz with constant $c_{2}$ (Statement 2), and also Proposition 5 holds.

It follows from Lemma 4 that the linear operators $\mathcal{L}^{ \pm}$resolving homological equations act from $\mathbf{B}_{m}\left(S_{R \varepsilon}\right)$ into $\mathbf{A}\left(S_{R \varepsilon}\right)$ and is bounded by the constant $\mathfrak{C}(m, \lambda)$. Hence, for each $f$ in $\mathbf{M}_{\omega}\left(S_{R \varepsilon}\right)$, the results of the action of the operator $\mathcal{R}[f]$ belongs to $\mathbf{B}_{m}\left(S_{R \varepsilon}\right)$ and the operators $\mathcal{H}^{ \pm}$satisfy the inequalities

$$
\left\|\mathcal{H}^{ \pm}[f]\right\|_{S_{R \varepsilon}} \leqslant\left\|\mathcal{L}^{ \pm}\right\|_{S_{R \varepsilon}} \cdot\|\mathcal{R}[f]\|_{S_{R \varepsilon}, m} \leqslant \mathfrak{C}(m, \lambda) \mathfrak{c}(R, m)
$$

for all $f \in \mathbf{M}_{\omega}\left(S_{R \varepsilon}\right)$. Thanks to the linearity of the operator $\mathcal{L}^{ \pm}$we also have:

$$
\left\|\mathcal{H}^{ \pm}\left[f_{1}\right]-\mathcal{H}^{ \pm}\left[f_{2}\right]\right\|_{S_{R \varepsilon}}=\left\|\mathcal{L}^{ \pm}\left(\mathcal{R}\left[f_{1}\right]-\mathcal{R}\left[f_{2}\right]\right)\right\|_{S_{R \varepsilon}} \leqslant \mathfrak{C}(m, \lambda) \mathfrak{c}(R, m)\left\|f_{1}-f_{2}\right\|_{S_{R \varepsilon}} .
$$

for all $f_{1,2} \in \mathbf{M}_{\omega}\left(S_{R \varepsilon}\right)$. By the choice of $R$ in Proposition 5 the relation holds:

$$
\mathfrak{c}(R, m) \mathfrak{C}(m, \lambda) \leqslant \omega \leqslant \frac{1}{2}
$$

Inequalities (26) and (28) imply that the operators $\mathcal{H}^{ \pm}$are well-defined and it follows from (27) and (28) that it is a contracting mapping. The proof is complete.

We construct the domains $S_{R \varepsilon}^{ \pm}$. We recall that $\delta \in\left(\frac{\pi}{4} ; \frac{\pi}{2}\right)$ and $\tan \delta>\frac{\lambda}{2 \pi}$. We also recall that $\Delta_{N}$ is the function in the right hand side in functional equations (5). The choice of the class $\mathbf{B}_{N-2}$ with the parameter $N-2$ is due to asymptotics (4).

Lemma 7. The following facts hold true on the sectorial domain $S_{R \varepsilon}$.

1. There exists a holomorphic bounded mapping $\tilde{H}_{N}^{ \pm}$adjoining $F_{0}$ with $\tilde{F}_{N}$ of the form:

$$
\tilde{H}_{N}^{ \pm}(\xi, z)=\left(\xi+h_{N}(\xi, z), z+g_{N}^{ \pm}(\xi, z)\right) ;
$$

2. $\tilde{H}_{N}^{ \pm}(\xi, z)=\left(\xi+o\left(|\xi|^{-N+6}\right), z+o\left(|\xi|^{-N+6}\right)\right)$ as $|\xi| \rightarrow \infty,(\xi, z) \in S_{R \varepsilon}^{ \pm}$;

3. The normalizing mapping adjoining $F_{0}$ with $\tilde{F}_{N}$ in the sectorial domain $S_{R \varepsilon}^{ \pm}$is unique in the class of holomorphic bounded functions obeying Statement 2.

Proof. The space $\mathbf{A}\left(S_{R \varepsilon}\right)$ equipped with norm 25$)$ is Banach and this is why the space $\mathbf{M}_{\omega}\left(S_{R \varepsilon}\right)$ with the induced metric is complete. Since by Lemma 6 the operator $\mathcal{H}^{ \pm}$acts from $\mathbf{M}_{\omega}\left(S_{R \varepsilon}\right)$ into $\mathbf{M}_{\omega}\left(S_{R \varepsilon}\right)$ and is contracting, by the contracting mapping principle, in the domain $S_{R \varepsilon}$ there exists unique $\left(h_{N}^{ \pm}, g_{N}^{ \pm}\right)$such that

$$
\left(h_{N}^{ \pm}, g_{N}^{ \pm}\right)=\mathcal{H}^{ \pm}\left(h_{N}^{ \pm}, g_{N}^{ \pm}\right)
$$

This implies that there exists the unique mapping $\tilde{H}_{N}^{ \pm}(\xi, z)=\left(\xi+h^{ \pm}(\xi, z), z+g_{N}^{ \pm}(\xi, z)\right)$ satisfying the pair of functional equations $(5)$, that is, the one adjoining $F_{0}$ with $\tilde{F}_{N}$. Since $\left(h_{N}^{ \pm}, g_{N}^{ \pm}\right) \in \mathbf{M}_{\omega}\left(S_{R \varepsilon}\right)$, then $\tilde{H}_{N}$ is holomorphic in $S_{R \varepsilon}$. This proves the first statement; the identity $h_{N}^{+}=h_{N}^{-}$on the intersection of $S_{R \varepsilon}^{+}$and $S_{R \varepsilon}^{-}$can be shown exactly in the same way as Proposition 3 was proved.

It follows from Lemma 5 that the operator $\mathcal{R}$ acts from $\mathbf{M}_{\omega}\left(S_{R \varepsilon}\right)$ into $\mathbf{B}_{m}\left(S_{R \varepsilon}\right)$ and hence, Lemmata 1 and 2 imply Statement 2.

Statement 3 can be proved similarly to Proposition 3. The proof is complete.

3.4. Proof of theorem on sectorial normalization in right half-neighbourhood. All arguing in this section are made under the choice of the parameters made in the proof of Lemma 7.

Existence of solution. Lemma 7 provides the holomorphic normalizing change of coordinates $\tilde{H}_{N}^{ \pm}$adjoining normal form $F_{0}$ with a preliminary normal form $\tilde{F}_{N}$ on a sectorial domain $S_{R \varepsilon}$. Then $\tilde{H}_{N}^{ \pm} \circ \widetilde{\mathrm{H}}_{N}$ is a holomorphic mapping adjoining $F_{0}$ with the mapping $\tilde{F}$ in $S_{R \varepsilon}$. 
Normalization. It follows from the construction that the mapping $\tilde{H}_{N}^{ \pm} \circ \tilde{\mathrm{H}}_{N}$ is normalized on $S_{R \varepsilon}^{ \pm}$.

We note that the normalization of the mapping $\tilde{H}_{N}^{ \pm} \circ \tilde{\mathrm{H}}_{N}$ in the sectorial domains $S_{R \varepsilon}^{ \pm}$implies their invertibility for a sufficiently large parameter $R$.

Uniqueness. Assume that there exist two holomorphic bounded and normalized mappings adjoining $F_{0}$ with $\tilde{F}$ on the domain $S_{R \varepsilon}^{ \pm}$. We denote them by $H^{ \pm}$and $G^{ \pm}$. The normalization implies the existence of the inverse mapping $\left(G^{ \pm}\right)^{-1}$, which acts from $G^{ \pm}\left(S_{R \varepsilon}^{ \pm}\right)$into $S_{R \varepsilon}^{ \pm}$. The mapping $\Phi^{ \pm}=H^{ \pm} \circ\left(G^{ \pm}\right)^{-1}$ is called a transition functions. It follows from the definition of the transition function that it commutes with normal form $F_{0}$ :

$$
F_{0} \circ \Phi^{ \pm}=F_{0} \circ H^{ \pm} \circ\left(G^{ \pm}\right)^{-1}=H^{ \pm} \circ \tilde{F} \circ\left(G^{ \pm}\right)^{-1}=H^{ \pm} \circ\left(G^{ \pm}\right)^{-1} \circ F_{0}=\Phi^{ \pm} \circ F_{0} .
$$

Let the transition function read as $\Phi^{ \pm}(\xi, z)=\left(\xi+h(\xi, z), z+g^{ \pm}(\xi, z)\right)$, be holomorphic, bounded and also be normalized. The commutation with the normal form implies that

$$
\begin{aligned}
& h(\xi, z)=h\left(\xi+1, e^{\lambda} z\right), \\
& e^{\lambda} g^{ \pm}(\xi, z)=g^{ \pm}\left(\xi+1, e^{\lambda} z\right) .
\end{aligned}
$$

We note that the derivative $\frac{\partial g^{ \pm}}{\partial z}$ satisfies the same relation as the function $h$ :

$$
\frac{\partial g^{ \pm}}{\partial z}(\xi, z)=\frac{\partial g^{ \pm}}{\partial z}\left(\xi+1, e^{\lambda} z\right)
$$

The Cauchy estimates [23] and the normalization imply that the derivative $\frac{\partial g^{ \pm}}{\partial z}$ is also holomorphic and bounded on probably smaller sectorial domain with a similar asymptotics.

We observe that equations $(30)$ and $(32)$ reproduces exactly equation $(11)$ in the proof of the uniqueness of the first homological equation in Lemma 1. Reproducing the same arguing, we obtain:

$$
h(\xi, z)=\frac{\partial g^{ \pm}}{\partial z}(\xi, z)=0, \quad(\xi, z) \in \mathbb{C}^{2}
$$

This implies that $g^{ \pm}$is independent of $z$ and for $g^{ \pm}(\xi, z)=g^{ \pm}(\xi)$ we have:

$$
e^{\lambda} g^{ \pm}(\xi)=g^{ \pm}(\xi+1), \quad \xi \in S_{\xi}^{ \pm} .
$$

Exactly the same equation in the domains $S_{\xi}^{ \pm}$has already been considered in the proof of the uniqueness of the reduced homological equation, see Proposition 4). This implies that $g^{ \pm} \equiv 0$ on $S_{\xi}^{ \pm}$.

Asymptotics. Let $\tilde{H}_{N}^{ \pm}$and $\tilde{\mathrm{H}}_{N}$ be the above constructed mappings. The above proven existence and uniqueness of the analytic continuation implies that $\tilde{H}^{ \pm} \stackrel{\text { def }}{=} \tilde{H}_{N}^{ \pm} \circ \tilde{\mathrm{H}}_{N}$ is independent of $N$. We note that in construction of $\tilde{\mathrm{H}}_{N}$ and $\tilde{H}_{N}$ the parameter $N>6$ can be chosen arbitrarily. Hence, since

$$
\tilde{H}_{N}^{ \pm}=i d+\left(O\left(|\xi|^{-N+6}\right), O\left(|\xi|^{-N+6}\right)\right), \quad|\xi| \rightarrow \infty,
$$

then for all $N>6$ we have

$$
\tilde{H}^{ \pm}=\tilde{\mathrm{H}}_{N}+O\left(|\xi|^{-N+6}\right), \quad|\xi| \rightarrow \infty .
$$

This yields that $\tilde{\mathrm{H}}$ is an asymptotic series for $\tilde{H}^{ \pm}$. 
End of the proof. We have constructed holomorphic sectorial normalizing mappings

$$
\tilde{H}_{N}^{ \pm}=\left(\xi+h_{N}(\xi, z), z+g_{N}^{ \pm}(\xi, z)\right)
$$

in sectorial domains

$$
S_{R \varepsilon}^{ \pm}=\left\{\xi: \operatorname{Re} \xi<-R<-1 ;-\delta< \pm(\pi-\arg \xi)<\frac{\pi}{2}\right\} \times\{|z|<\varepsilon\} .
$$

We note that the functions $h_{N}$ and $g_{N}^{ \pm}$satisfy functional equations (5).

We continue $h_{N}$ and $g_{N}^{ \pm}$on sectorial domains

$$
\tilde{S}_{R \varepsilon}^{ \pm}=\left\{\xi:|\xi|>R>1 ;-\delta< \pm(\pi-\arg \xi)<\frac{\pi}{2}\right\} \times\{|z|<\varepsilon\} .
$$

by the functional equations. We denote $\xi_{j}=\xi-j, z_{j}=e^{-\lambda j} z$. Then for each $\xi \in \tilde{S}_{R \varepsilon}^{ \pm} \backslash S_{R \varepsilon}^{ \pm}$ there exists $k=[\operatorname{Re} \xi-R+1]$ such that $\left(\xi_{k}, z_{k}\right) \in S_{R \varepsilon}^{ \pm}$. Then $h_{N}$ and $g_{N}^{ \pm}$can be well continued by induction to holomorphic and bounded on $\tilde{S}_{R \varepsilon}^{ \pm}$functions by means of the formulae:

$$
\begin{aligned}
& h_{N}(\xi, z)=h_{N}\left(\xi_{k}, z_{k}\right)+\sum_{n=1}^{k} \Delta_{1 N}\left(\xi_{n}+h_{N}\left(\xi_{n}, z_{n}\right), z_{n}+g_{N}\left(\xi_{n}, z_{n}\right)\right), \\
& g_{N}(\xi, z)=e^{\lambda k} g_{N}\left(\xi_{k}, z_{k}\right)+\sum_{n=1}^{k} e^{\lambda(n-1)} \Delta_{1 N}\left(\xi_{n}+h_{N}\left(\xi_{n}, z_{n}\right), z_{n}+g_{N}\left(\xi_{n}, z_{n}\right)\right) .
\end{aligned}
$$

It follows from the estimates for $\tilde{H}_{N}$ on $S_{R \varepsilon}$ and the boundedness of $k$, which exceeds $R$, that the continued in this way on $\tilde{S}_{R \varepsilon}$ mapping is unique, holomorphic, bounded and preserves its asymptotic properties on $\tilde{S}_{R \varepsilon}^{ \pm}$.

Choosing the parameters of the domains $\tilde{S}_{R \varepsilon}^{ \pm}$large enough, namely, the radius $R$ and opening $\delta$, and choosing the radius $\varepsilon$ small enough, without loss of generality we can assume that the preimage of the left upper (left lower) sectorial domain $\tilde{S}_{R \varepsilon}^{+}$(respectively, $\tilde{S}_{R \varepsilon}^{-}$) under the mapping $B:(x, y) \mapsto\left(\xi=-\frac{1}{x}, z=y\right)$ is the right upper sectorial domain $\Omega^{+}$(respectively, $\Omega^{-}$), and the constructed mapping $H^{ \pm}=B^{-1} \circ \tilde{H}^{ \pm} \circ B$ satisfies Theorem 1. This completes the proof of the theorem.

\section{BIBLIOGRAPHY}

1. H. Poincaré. Mémoire sur les courbes définies par une équation différentielle I // J. Math. Pures Appl. 7, 375-422 (1881).

2. H. Poincaré. Mémoire sur les courbes définies par une équation différentielle II // J. Math. Pures Appl. 8, 251-296 (1882).

3. H. Poincaré. Sur les courbes définies par les équations différentielles III // J. Math. Pures Appl. 1, 167-244 (1885).

4. H. Poincaré. Sur les courbes définies par les équations différentielles IV // J. Math. Pures Appl. 2, 151-218 (1886).

5. H. Poincaré Sur les problém des trois corps et les équations de la dinamique // Acta Math. 13, 1-271 (1890).

6. C.L. Siegel. Vorlesungen uber Himmelsmechanik. Springer, Berlin (1957).

7. A.D. Bryuno. The analytic form of differential equations // Trudy Mosk. Matem. Obsch. 25, 119-262 (1971). (in Russian).

8. A.D. Bryuno. The analytic form of differential equations // Trudy Mosk. Matem. Obsch. 26, 199-239 (1972). (in Russian).

9. V.I. Arnold. Supplementary chapters to the theory of ordinary differential equations. Nauka, Moscow (1978). (in Russian).

10. J.C. Yoccoz. Linéarisation des germes de difféomorphismes holomorphes de (C,0)// C.R. Acad. Sci. Paris Sér. I Math. 306:1, 55-58 (1988). 
11. S.M. Voronin. Analytic classification of germs of conformal mappings $(\mathbb{C}, 0) \rightarrow(\mathbb{C}, 0)$ with identity linear part // Funkts. Anal. Pril. 15:1, 1-17 (1981). [Funct. Anal. Appl. 15:1, 1-13 (1981).]

12. J. Ecalle. Les fonctions resurgentes. Publ. Math. d'Orsay, Orsay (1981).

13. J. Martinet, J.P. Ramis. Problème de modules pour des équations différentielles non linéaires du premier ordre// Inst. Hautes Études Sci. Publ. Math. 55, 63-164 (1982).

14. J. Martinet, J.P. Ramis. Classification analytique des équations différentielles non linéaires resonnantes du premier ordre // Ann. Sci. École norm. supér. 16:4, 571-621 (1983).

15. Y.S. Il'yashenko. Nonlinear Stokes phenomena // in "Nonlinear Stokes Phenomena". Adv. Sov. Math. 14, 1-55 (1992).

16. S.M. Voronin. Darboux-Whitney's problem and related questions // in "Nonlinear Stokes Phenomena". Adv. Sov. Math. 14, 139-223 (1992).

17. Yu.S. Ilyashenko, S.Yu. Yakovenko. Analytic theory of differential equatinos. V. 1. MCCME, Moscow (2013). (in Russian).

18. S.M. Voronin. Analytic classification of germs of holomorphic mapping with non-isolated equilibria and constant multiplications and applications // Vestnik ChelGU. 5, 12-30 (1999). (in Russian).

19. P.A. Shaikhullina. Formal normalization of generic germs for semihyperbolic maps// Matem. Zamet. SVFU. 22:4, 79-90 (2015). (in Russian).

20. S.M. Voronin, P.A. Fomina. Sectorial normalization of germs of semi-hyperbolic mappings // Vestnik ChelGU. 16, 94-113 (2013). (in Russian).

21. T. Ueda. Local structure of analytic transformations of two complex variables, I// J. Math. Kyoto Univ. 26:2, 233-261 (1986).

22. T. Ueda. Local structure of analytic transformations of two complex variables, II // J. Math. Kyoto Univ. 31:3, 695-711 (1991).

23. B.V. Shabat. Introduction into complex analysis. Nauka, Moscow (1985). (in Russian).

24. P. A. Shaikhullina. On solution to the simplest functional equation in curvilinear-band-type domain // Matem. Zamet. SVFU. 24:4, 87-95 (2017). (in Russian).

Polina Alexeevna Shaikhullina

Chelyabinsk State University,

Br. Kashiriny str. 129,

454001, Chelyabinsk, Russia

E-mail: fominapa@gmail.com 